Retraction

\title{
Retracted: A Comparison between Three-Dimensional Visualization Guided Hepatectomy and Ultrasonography Guided Radiofrequency Ablation in the Treatment of Small Hepatocellular Carcinoma within the Milan Criteria
}

\author{
BioMed Research International \\ Received 10 April 2017; Accepted 10 April 2017; Published 7 June 2017 \\ Copyright (C) 2017 BioMed Research International. This is an open access article distributed under the Creative Commons \\ Attribution License, which permits unrestricted use, distribution, and reproduction in any medium, provided the original work is \\ properly cited.
}

At the request of the authors, the article titled "A Comparison between Three-Dimensional Visualization Guided Hepatectomy and Ultrasonography Guided Radiofrequency Ablation in the Treatment of Small Hepatocellular Carcinoma within the Milan Criteria" [1] has been retracted. The article was found to contain substantial errors, as detailed below, which mean the data do not support the conclusions. The authors apologize for these errors.

(1) Nine patients were wrongly classified. Five patients in the surgery group with mixed hepatocellular carcinoma, cholangiocarcinoma in the postoperative pathological analysis, were wrongly recorded as having hepatocellular carcinoma based on intraoperative frozen section examination. Four patients in the radiofrequency ablation group had severe dysplasia rather than hepatocellular carcinoma according to histopathology in the follow-up period.

When these patients are excluded, liver function is a risk factor for survival (hazard ratio $=1.8, p=0.015$ ), but this parameter was wrongly excluded from the risk factors in the published article.

(2) There was high multicollinearity between liver function of Child-Pugh Class and Tbil, Alb, and PT (eigenvalue $=0.07 \approx 0$, condition index $=20.484>10$; Table 1 ). Due to this, the results may change unpredictably in response to small changes in the model or the data [2]. To overcome this issue, we need a larger sample size and improved statistical methodologies [3]. 
TABLE 1: Results of the collinearity diagnostics.

\begin{tabular}{|c|c|c|c|c|c|c|c|}
\hline \multicolumn{8}{|c|}{ Collinearity diagnostics $^{\mathrm{a}}$} \\
\hline \multirow{2}{*}{ Model } & \multirow{2}{*}{ Dimension } & \multirow{2}{*}{ Eigenvalue } & \multirow{2}{*}{ Condition index } & \multicolumn{4}{|c|}{ Variance proportions } \\
\hline & & & & (Constant) & Tbil & ALB & $\mathrm{PT}$ \\
\hline \multirow[t]{4}{*}{1} & 1 & 2.972 & 1.000 & 0.00 & 0.02 & 0.00 & 0.02 \\
\hline & 2 & 0.845 & 1.876 & 0.00 & 0.01 & 0.00 & 0.97 \\
\hline & 3 & 0.177 & 4.103 & 0.01 & 0.91 & 0.02 & 0.00 \\
\hline & 4 & 0.007 & 20.484 & 0.99 & 0.06 & 0.98 & 0.01 \\
\hline
\end{tabular}

${ }^{a}$ Dependent variable: Child-Pugh.

\section{References}

[1] T.-P. Guan, C.-H. Fang, J. Yang, N. Xiang, Q.-S. Chen, and S.-Z. Zhong, "A comparison between three-dimensional visualization guided hepatectomy and ultrasonography guided radiofrequency ablation in the treatment of small hepatocellular carcinoma within the Milan criteria," BioMed Research International, vol. 2016, Article ID 8931732, 2016.

[2] P. Claret, X. Bobbia, and J. E. de La Coussaye, "Collinearity and multivariable analysis," Intensive Care Medicine, vol. 42, no. 11, pp. 1834-1834, 2016.

[3] Y.-K. Tu, M. Kellett, V. Clerehugh, and M. S. Gilthorpe, "Problems of correlations between explanatory variables in multiple regression analyses in the dental literature," British Dental Journal, vol. 199, no. 7, pp. 457-461, 2005. 